\title{
BMJ Open Screening nursing students to identify those at high risk of poor mental health: a cross-sectional survey
}

\author{
Der-Yun Hsiung, ${ }^{1,2}$ Ching-Lun Tsai, ${ }^{1}$ Ling-Chun Chiang, ${ }^{2}$ Wei-Fen Ma ${ }^{3,4,5}$
}

To cite: Hsiung D-Y, Tsai C-L, Chiang L-C, et al. Screening nursing students to identify those at high risk of poor mental health: a crosssectional survey. BMJ Open 2019;9:e025912. doi:10.1136/ bmjopen-2018-025912

- Prepublication history for this paper is available online. To view these files, please visit the journal online (http://dx.doi. org/10.1136/bmjopen-2018025912).

Received 8 August 2018 Revised 11 April 2019 Accepted 28 May 2019

Check for updates

(C) Author(s) (or their employer(s)) 2019. Re-use permitted under CC BY-NC. No commercial re-use. See rights and permissions. Published by BMJ.

${ }^{1}$ Department of Public Health, China Medical University,

Taichung, Taiwan

${ }^{2}$ Department of Nursing, HungKuang University, Taichung, Taiwan

${ }^{3}$ School of Nursing, China

Medical University, Taichung,

Taiwan

${ }^{4}$ Department of Nursing, China Medical University Hospital,

Taichung, Taiwan

${ }^{5}$ Brain Disease Research Center, China Medical University Hospital, Taichung, Taiwan

Correspondence to

Professor Wei-Fen Ma;

Indaisy@mail.cmu.edu.tw

\begin{abstract}
Objectives This study aimed to identify those at high risk of poor mental health among nursing students and to examine the relationships and consistency among five mental health assessments.
\end{abstract}

Design A cross-sectional design with purposive sampling was used. Four mental health screening tools plus previous mental health history/information were conducted during June 2015 to October 2016.

Setting A nursing major university in Taiwan.

Participants A total of 2779 participants aged between 19 and 45 years were recruited.

Main outcome measures Five mental health risks were identified: increased risk for ultra-high risk, putative prepsychosis states, high trait anxiety, high state anxiety with genetic risk and depression.

Results Out of the 3395 collected questionnaire, 2779 (82\%) were found valid and included in the study. $612(22 \%)$ of the participants were identified to be at mental health risk. 12 of them appeared positive in four, 79 in three, 148 in two and 373 in one of the adopted mental health screening tools. 69 participants had the experience of seeking medical help due to mental health issues, and $58 \%$ of them were identified to be at risk of mental health during the screening. Trait and state anxiety scored the highest in correlation analysis $(r(2620)=0.76, p<0.001)$. Correlations were observed in the scores on the subscales of Chinese Version of Schizotypal Personality Questionnaire-Brief (CSPQ-B) and those of Brief self-report Questionnaire for Screening Putative Prepsychotic States (BQSPS), and the highest correlation coefficients was $(r(2740)=0.70, p<0.001)$. Although both of the screening tools are used to assess mental illness risk at its prodromal phase, but CSPQ-B in general appeared to have more rigorous screening criteria than BQSPS.

Conclusion For expediting early identification high risk of poor mental health, easy-to-use screening questionnaires can be adopted to assess the mental health state of nursing students whose mental well-being and overall health are of vital importance to the entire healthcare industry.

\section{INTRODUCTION}

The onset of most psychotic disorders tend to occur between ages 15 and 30 years and approximately three-quarters of mental disorders begin before age 24 years. ${ }^{1}$ Sixty-five per cent of at-risk young adults have tendency to deny any current mental health problems and view help seeking to be negative, thus failing to seek

\section{Strengths and limitations of this study}

To the best of our knowledge, this study was the first one to use four mental health screening tools plus demographic data including personal and family history of mental illness for assessing anxiety, depression and other symptoms of mental health among nursing students. As such, the study builds its strength in the comprehensive depth and breadth of its investigation on the mental status of nursing students.

- It should be noted that the study relied on self-reported questionnaires which were non-clinical sample and no second-phase clinical interviews had been conducted. False positive is therefore an issue demanding attention.

- For the nursing students identified by the study to be at risk of poor mental health, clinical interviews should be arranged as follow-ups for monitoring and developing appropriate interventions.

professional assistance. ${ }^{2}$ Poorer prognosis of psychotic disorders and treatment effectiveness had been found to be associated with the longer duration of untreated psychosis (DUP). ${ }^{3}$ Early identification and intervention is therefore highly crucial in delaying or even avoiding the onset of disease and mitigating its subsequent long-term damage. ${ }^{4}$

Studies paid the attention to the mental health screening of college students and young community dwellers and showed a detection rate ranging $3.5 \%-8.1 \% .^{5}$ Twenty-six to thirty-seven per cent of teenagers and college students susceptible to "ultrahigh risk' were accompanied with anxiety and depression. ${ }^{5-7}$ Ultra-high risk was first introduced by Yung and McGorry in $1996 .{ }^{8}$ It refers to the manifestation of the brief limited intermittent or attenuated psychotic symptoms or functional decline that influences the psychosocial function and the quality of life. Ultra-high risk cases have symptoms similar to the prodromal period of mental illness, but have not yet reached the diagnostic criteria of the disease. ${ }^{8}$ Their transition rates range 
between $10 \%$ and $50 \%$ over a 2 -year to 5 -year period. ${ }^{9}$ As these young people have yet to establish a solid foundation in social, educational and occupation competence, symptoms are more likely to inflict greater damage along longer DUP. ${ }^{10}$ Assessment and identification for those at high risk of poor mental health and subsequent intervention and treatment prior to the onset of disease should therefore be regarded as a key to the prevention of mental illness. ${ }^{10}$

On the cusp of transition from adolescence to adulthood, many college students need to cope with significant changes in lives-moving away from home, taking responsibility for themselves, balancing studies and social life, and getting prepared for future career-all of which can be stressors jeopardising their mental health. ${ }^{2}$ According to a previous study, three-fourths of college had experienced nervousness, anxiety, depression and other emotional problems during the last year. ${ }^{11}$ In addition, anxiety, depression, psychotic disorders, eating disorders, self-harm and obsessive-compulsive disorders have been identified as the most common mental issues troubling college students in a systematic literature review. ${ }^{12}$ The current mental state of an individual is closely and directly related to his or her future well-being. Thus, the mental health of college students is an increasingly important issue. ${ }^{13}$

In 2018, an Irish study on the prevalence of depressive symptoms and suicidal ideation among first year undergraduate university students found that $59 \%$ of participants experienced depressive symptoms and $28.5 \%$ had suicidal ideation. ${ }^{14}$ Another systematic review and integration analysis in 2013 showed that the average depressive symptoms prevalence rate of general college students was $30.6 \%{ }^{15}$ Nursing students were higher than the average (34\%) especially in Asia (43\%). ${ }^{16}$ Approximately $21 \%-23 \%$ of the nursing students in Taiwan and mainland China have moderate to severe depression. ${ }^{1718}$

Financial stress and poor relationships with both parents, academic performance, academic stress, not having clear college goals, interest in majors, occupational future (including manageability of clinical work) and alcohol consumption increased the odds of experiencing the mental health symptoms. ${ }^{1314} 18$ Nursing students are more vulnerable to stress, anxiety and depression than non-nursing or non-medicine majors because of their challenges in difficult specialty, skills and clinical praticum. ${ }^{19}$ The nursing professional responsibilities require a higher moral standard and more rigorous ethical principles. ${ }^{16} 19$ The mental health of nursing students is therefore an issue meriting close attention and in-depth examination. Nowadays, although various mental health screening instruments are available, there still has been a lack of empirical evidence to compare their effectiveness.

This study investigated to identify those at high risk of poor mental health among college nursing students with a focus on ultra-high risk symptoms, depression and anxiety and examined the consistency of the five adopted mental health assessment and screening tools. This study has the following three hypotheses:
1. The numbers of the mental health risks students identified by each of the four mental health screening tools have no differences statistically.

2. The scores of the four mental health screening tools are not statistically correlated.

3. There are no significant differences between male and female on the scores of the four mental health screening tools.

\section{METHOD}

\section{Research design and participants}

A cross-sectional design was used for this study. Three thousand two hundred and twenty-five nursing majors (in the Bachelor of Science in Nursing (BSN) programme of a university in central Taiwan) and 170 nursing students (fourth- year and fifth-year ones) in the university's 5-year junior college programme were recruited through purposive sampling. The study followed the inclusion criteria of Kuang Tien General Hospital Institutional Review Board. The participants were 18 years and older nursing major students with written consent. The researchers' mentorship classes and the minor students under the age of 18 years were excluded from the study.

\section{Data collection}

Recruitment of participants was conducted during the period from June 2015 to October 2016. The academic advisers or class leaders were requested to gather prospective participants in the meetings, and the study team attended the meetings to explain the objective and procedure of the study and the rights of the participants. Questionnaires were issued to students who expressed their willingness of participation in written consents. All the questionnaires were administered together in the sequence of (1) Chinese Mandarin State-Trait Anxiety Inventory Y (CMSTAI-Y); (2) Chinese Version of Schizotypal Personality Questionnaire-Brief (CSPQB); (3) Taiwanese Depression Questionnaire (TDQ); (4) Brief self-report Questionnaire for Screening Putative Prepsychotic States (BQSPS); (5) previous mental health history/information. Questionnaires took $\sim 20-25 \mathrm{~min}$, and the survey ended with the participants' submitting the completed questionnaires to the study team. Figure 1 showed the flowchart of participant enrolment and questionnaire collection.

\section{Patient and public involvement}

Patient and public involvement was not sought in the design of this study nor in the development of the research questions. Patient involvement in the recruitment and conduct of this study is described in the research design and participants and data collection of this paper. Participants were asked during the recruitment stage if they want to be informed about the study findings. We would disseminate our findings to those who expressed interests in our findings by contacting researcher's mobile phone. 


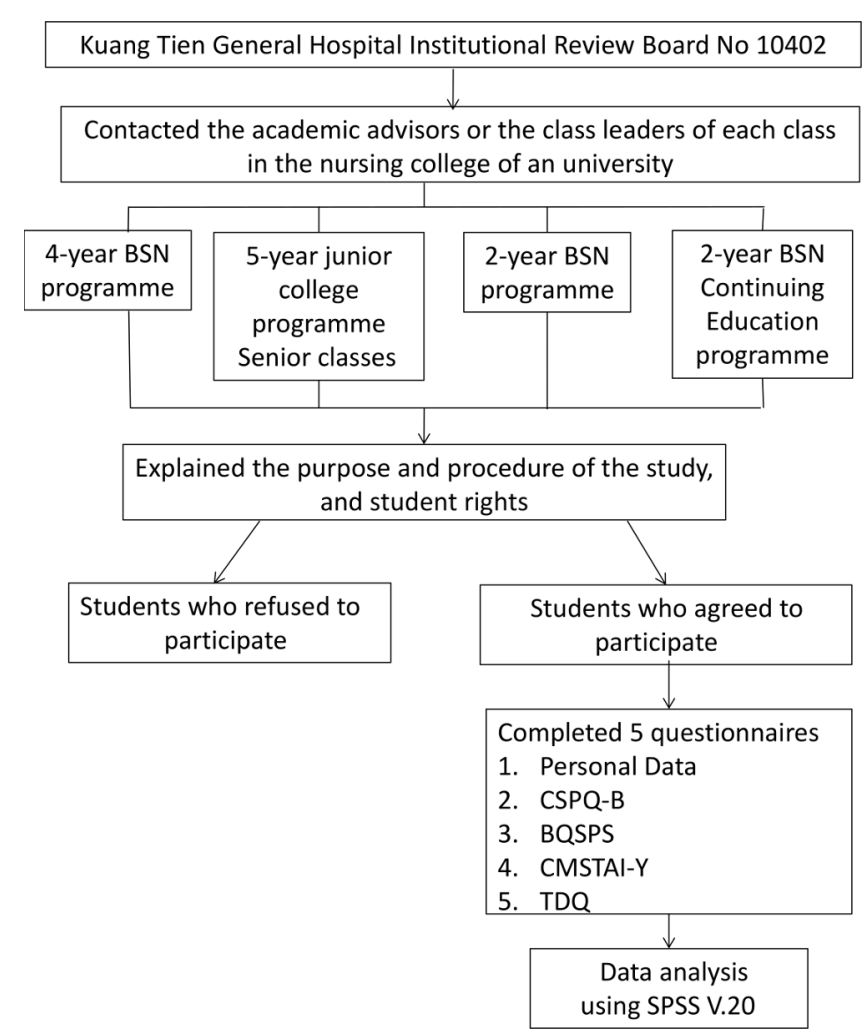

Figure 1 Flowchart of participant enrolment and questionnaire collection. BQSPS, Brief self-report Questionnaire for Screening Putative Prepsychotic States; CMSTAI-Y, Chinese Mandarin State-Trait Anxiety Inventory Y; CSPQ-B, Chinese Version of Schizotypal Personality Questionnaire-Brief; TDQ, Taiwanese Depression Questionnaire.

\section{Instruments}

Keshavan et al proposed that there are multiple pathways affecting the risk factors of mental high risk and the subsequent development of the symptoms are (1) family/ genetic risk, (2) psychological traits (such as schizotypal personality traits) and (3) symptoms expression. ${ }^{20}$ This study assessed the family history and family/genetic risk in previous mental health history/information. The personality traits were assessed by Schizotypal Personality Questionnaire-Brief (SPQ-B). The symptomatic measures included suspected prepsychiatric symptoms were assessed by BQSPS. Anxiety were assessed by CMSTAI-Y. And depression were assessed by TDQ.

\section{Previous mental health history/information}

Previous mental health history/information incorporates 12 questions for obtaining essential information of the participants, including family name, gender, age, contact numbers, education background, marriage status, religious belief, ages of parents at the time of the participant's birth, personal history of mental illness, personal medical history, family history of mental illness and self-assessment of sleep quality.
Chinese Version of Schizotypal Personality Questionnaire-Brief SPQ-B was developed by Raine and Benishay. ${ }^{21}$ It is a self-report scale covers 22 true-false questions divided into the following three subscales: the 8-question interpersonal subscale, the 8-question cognitive-perceptual subscale and the 6-question disorganisation subscale. A higher score indicates a more severe degree of deficit. The 2-month test-retest reliability and the internal consistency of SPQ-B were estimated to be, respectively, 0.86 0.95 and 0.72 0.80. ${ }^{21}{ }^{22} \mathrm{SPQ}-\mathrm{B}$ was translated into Chinese ${ }^{23}$ and reported the following results: 2-week test-retest reliability 0.82 , internal consistency 0.76 , maximum value of receiver operating characteristic (ROC) curve 0.83, optimal score 17 points (with the maximum OR set at 24.4), sensitivity 0.80 and specificity $0.86 .{ }^{23}$ In this study, the Cronbach's coefficient for the CSPQ-B (22 items) was 0.82. It is therefore obvious that CSPQ-B has been proved to be a valid and reliable screening instrument that can be recommended for assessing psychosis risk. ${ }^{23}$

Brief self-report Questionnaire for Screening Putative Prepsychotic States

BQSPS was developed by Liu $e t a l^{24}$ It incorporated 15 true-false questions based on a four-factor structure: 5 questions are related to interpersonal difficulty/social anxiety, 4 to subthreshold psychotic-like experiences, 3 to self-depreciation and 3 to negative symptoms. A higher score indicates a more severe degree of deficit. BQSPS echoed the four-factor structured interview for prodromal syndrome and reported a sensitivity of 0.78 and a specificity of 0.71 . A screened individual answering 'true' to more than eight questions or to more than three questions covering any of question 1, 2 or 15 is deemed to be at a putative prepsychotic state (PPS).$^{24}$ The scale is also applicable to non-clinical samples for screening psychotic-like experiences and PPS. In this study, the Cronbach's coefficient for the BQSPS (15 items) was 0.83 . BQSPS had its validity and reliability tested ${ }^{25}$ and been applied to screen college students. ${ }^{26}$

\section{Chinese Mandarin State-Trait Anxiety Inventory Y}

The original English version of State and Trait Anxiety Inventory Y Form (STAI-Y) was revised by Spielberger $e t$ $a l$ in 1983 from the X Form, incorporating two subscales focusing, respectively, on state anxiety (STAI-Y1) and trait anxiety (STAI-Y2). Each subscale includes 20 self-report items for measuring state-trait anxiety on a 4-point Likerttype scale. The total score on each subscale ranges from 20 to 80 with a score exceeding 60 points indicating a high level of anxiety. ${ }^{27}$ The inventory was translated into Chinese by Ma et al and tested on 306 patients with anxiety disorders. The Cronbach's $\alpha$ read 0.91 (state) and 0.92 (trait) and the 2-week test-retest reliability read 0.76 (state) and 0.91 (trait), indicating fine internal consistency and stable reliability. ${ }^{28}$ Its high correlation with the Chinese version of the Hamilton Anxiety Rating Scale (0.69 for state and 0.74 for trait) and four-factor structure suggest decent construct validity. ${ }^{28}$ The internal 
consistency of Chinese Mandarin State-Trait Anxiety Inventory Y1 (CMSTAI-Y1) score (20 items) was 0.94 and Chinese Mandarin State-Trait Anxiety Inventory Y2 (CMSTAI-Y2) (20 items) was 0.91 in the present study.

\section{Taiwanese Depression Questionnaire}

TDQ was developed by Lee and colleagues. ${ }^{29}$ It emphasises the verbal characteristics of Taiwanese people covers 18 questions based on a three-factor structure for measuring depression during the last week. Five questions are related to emotion, six to physical condition and seven to cognition. A 4-point (0 3) Liker-type scale is used with the total score ranging from 0 to 54 points. Its Cronbach's $\alpha$ read 0.90 , the area under ROC curves 0.92 , the optimal cut-off point 19, sensitivity 0.89 and specificity 0.92 . A score $\geqq 19$ points suggests a state of depression requiring intervention or treatment, and a score $\geqq 29$ points indicates major/severe depression. The internal consistency reliability of TDQ (18 items) in this study was good (Cronbach's $\alpha=0.92)$. Culturally specific wording is used to make TDQ a culturally relevant instrument with satisfactory validity and reliability for screening depressive people in local communities. ${ }^{29}$

\section{Main outcome measures}

The study adopted four screening instruments and previous mental health history/information covering individual and family history of mental illness to assess the mental status of nursing students in Taiwan, focusing on the identification of the following five risks to poor mental health:

1. Risk 1: increased risk for ultra-high risk (IRUHR). An individual is at increased risk for ultra-high risk when he or she manifests brief limited intermittent or attenuated psychotic symptoms or functional decline and behavioural changes that affect his or her mental, social, educational and occupation competence, as well as quality of life. A participant scoring $\geqq 17$ points on CSPQ-B in the study was considered a IRUHR case at a heightened risk for developing ultra-high risk symptoms. ${ }^{23}$

2. Risk 2: PPS. An individual is marked with PPS if he or she manifests cognitive deficits or affective symptoms or undergoes interpersonal difficulties, social isolation, school failure and other subthreshold psychotic-like experiences. A participant scoring $\geqq 8$ points on BQSPS in the study was considered a PPS case. ${ }^{24}$

3. Risk 3: high trait anxiety (HTA). An individual is considered to have HTA if he or she manifests a relatively stable disposition to anxiety, experiencing apprehension, nervousness, worry or stress on a long-tern stable condition. A participant scoring $\geqq 60$ points on CMSTAI-Y2 in the study was regarded as a HTA case. ${ }^{28}$

4. Risk 4: high state anxiety with genetic risk (HSAGR). An individual may have HSAGR if he or she experiences feelings of non-specific stress, threat and uneasiness causing impermanent anxiety and with genetic risk form mental illness. A participant in the study scoring $\geqq 60$ points on CMSTAI-Y1 and with a first-degree relative having a history of mental illness was considered a HSAGR case at a heightened risk for developing mental health disorders. ${ }^{28}$

5. Risk 5: depression. An individual is immersed in depression when his or her physical, emotional and cognitive experiences during the recent 1 week show signs of depression. A participant scoring $\geqq 19$ points on TDQ in the study was considered to have moderate or severe depression. ${ }^{29}$

\section{Statistical analysis}

SPSS V.20.0 was used for data analysis on the percentages, means, SD and medians. The Pearson correlation coefficients were used for measuring the correlation between the variables. ${ }^{30}$ The statistical significance level was set at 0.05 . Priori power / sample size was estimated by $G^{*}$ power 3.1 .9 , the sample size needed was 1180 (power $=95 \%$, $\alpha=0.05$, effect size $=0.1$ and proportion of estimated incomplete $/$ missing data $=10 \%) .{ }^{31}$ Based on the criteria of the adopted mental health screening tools, this study focused on identifying the following five high risk of poor mental health among the recruited nursing students: IRUHR, PPS, HTA, HSAGR and depression. And the agreement between the five definitions high risk of poor mental health was determined using the kappa statistic.

\section{RESULTS}

\section{Personal data}

A total of 3395 questionnaires were issued and 2940 were responded. In that, 2779 (82\%) valid questionnaires from participants aged between 19 and 45 years with an average age of 22.99 years $(\mathrm{SD}=3.05)$ were included for the analysis. The participants were nursing students ranging from 5-year junior college programme, 4-year BSN programme, 2-year BSN programme and 2-year continuing education programme. There were 1642 nursing students recruited in 2015 and 1137 recruited in 2016, with a total of 2779 recruitments. Table 1 shows the personal data of the participants. The incomplete/ missing data were eliminated from table 1 . There were 69 (3\%) participants reported the experience of seeking medical help for mental health issues including depression $(23 / 69)$, anxiety disorder $(10 / 69)$, sleep disorder $(10 / 69)$, autonomic nerve dysfunction $(3 / 69)$, post-traumatic stress disorder (2/69), adjustment disorder $(1 / 69)$, bipolar disorder (1/69) and eating disorder (1/69); and 20 of the 69 participants chose not to specify the issue. Furthermore, there were 385 (14\%) participants having family members with a history of mental illness including schizophrenia $(50 / 385)$, bipolar disorder (94/385), depression (217/385), anxiety disorder (21/385) and others. The family members were reported as parents for 182 participants, siblings for 27 participants, grandparents for 62 participants, and uncles, aunts, and cousins for 156 participants. In respect of quality of sleep, 779 (28\%) participants experienced various types of sleep 


\begin{tabular}{|c|c|c|}
\hline Item & $\mathbf{n}$ & $\%$ \\
\hline \multicolumn{3}{|l|}{ Gender } \\
\hline Male & 201 & 7.3 \\
\hline Female & 2542 & 92.7 \\
\hline \multicolumn{3}{|l|}{ Age (years) } \\
\hline$>20$ & 116 & 4.4 \\
\hline $20-24$ & 2127 & 82.6 \\
\hline $25-29$ & 218 & 9.0 \\
\hline$\geqq 30$ & 107 & 4.0 \\
\hline \multicolumn{3}{|l|}{ Education } \\
\hline 5-Year junior college & 166 & 6.0 \\
\hline 4-Year BSN & 834 & 30.0 \\
\hline 2-Year BSN & 1124 & 40.4 \\
\hline 2-Year continuing education & 655 & 23.6 \\
\hline \multicolumn{3}{|l|}{ Religious belief } \\
\hline NIL & 1395 & 51.4 \\
\hline Buddhism & 228 & 8.4 \\
\hline Folk Taoism & 863 & 31.7 \\
\hline Christianity/Catholicism & 159 & 5.8 \\
\hline I-Kuan Tao & 66 & 2.4 \\
\hline Others & 8 & 0.3 \\
\hline \multicolumn{3}{|c|}{ Experience of seeking medical help for mental issues } \\
\hline No & 2669 & 97.5 \\
\hline Yes & 69 & 2.5 \\
\hline
\end{tabular}

Family members with history of mental illness

\begin{tabular}{|c|c|c|}
\hline No & 2359 & 86 \\
\hline Yes & 385 & 14 \\
\hline \multicolumn{3}{|l|}{ 3-Month quality of sleep } \\
\hline Fine & 1963 & 71.6 \\
\hline Difficulty falling asleep & 424 & 15.5 \\
\hline Sleep disruption & 281 & 10.2 \\
\hline Insomnia & 57 & 2.1 \\
\hline Reliance on sleep medications & 17 & 0.6 \\
\hline
\end{tabular}

problems during the last 3 months and $17(1 \%)$ were on sleep medications. In the study population, male only scored higher in CSPQ-B $(\mathrm{M}=5.94, \mathrm{SD}=4.51)$ than female $(\mathrm{M}=5.03, \mathrm{SD}=3.95), \mathrm{t}(217)=2.75, \mathrm{p}=0.006$. No other significant differences were found in other screening tools.

\section{Major mental health risks}

This study focused on the identification for the five high risk of poor mental health: IRUHR, PPS, HTA, HSAGR and depression. Of the 2779 participating nursing students, $612(22 \%)$ were found at risk of developing at least one major mental health risk, and 2167 (78\%) were reported having no significant sign of risk. Out of the 612 at risk individuals, $12(<1 \%)$ and $79(3 \%)$ participants appeared to have respectively four and three of the five
Table 2 Study outcomes of mental health risks and helpseeking behaviour $(n=2779)$

\begin{tabular}{lllll}
\hline $\begin{array}{l}\text { Types of mental } \\
\text { health risks }\end{array}$ & $\begin{array}{l}\text { Number } \\
(\mathbf{n})\end{array}$ & $\mathbf{( \% )}$ & $\begin{array}{l}\text { No of help- } \\
\text { seekers }\end{array}$ & $\begin{array}{l}\text { \% of help- } \\
\text { seekers }\end{array}$ \\
\hline IRUHR & 33 & 1.2 & 4 & 12.1 \\
\hline PPS & 446 & 16.0 & 26 & 5.8 \\
\hline HTA & 127 & 4.6 & 13 & 10.2 \\
\hline HSAGR & 6 & 0.2 & 1 & 16.7 \\
\hline Depression & 342 & 12.3 & 34 & 9.9
\end{tabular}

No of risks identified

\begin{tabular}{lllll}
0 & 2167 & 78.0 & 29 & 1.3 \\
1 & 373 & 13.4 & 16 & 4.3 \\
2 & 148 & 5.3 & 12 & 8.1 \\
3 & 79 & 2.8 & 10 & 12.7 \\
4 & 12 & 0.4 & 2 & 16.7 \\
\hline
\end{tabular}

0 , identified with zero targeted risk to mental illness.

1 , identified positive for one of the five targeted risks.

2 , identified positive for two of the five targeted risks.

3 , identified positive for three of the five targeted risks.

4 , identified positive for four of the five targeted risks HSAGR, high state anxiety with genetic risk; HTA, high trait anxiety; IRUHR, increased risk for ultra-high risk; PPS, putative prepsychosis states.

mental health risks. PPS emerged to be the most common risk as identified in $446(16 \%)$ participants, followed by depression (342 participants and 12\%).

Of the $69(3 \%)$ participants with the experience of seeking medical help for mental health issues, $40(58 \%)$ of them were identified to be at risk of mental health during the screening. Among them, 2 were having four of the risks, 10 were at three and 12 at two. There were one $(17 \%)$ of the participants identified to be positive for HSAGR and four (12\%) of participants positive for IRUHR had the experience of seeking medical help for their mental problems. Based on the study results, participants identified to have a greater number of the mental health risks tended to be more likely to seek medical assistance. Around 2 (17\%) of those with four risks and $10(13 \%)$ of those with three risks used to seek medical assistance. Participants identified with no risk reported the lowest percentage of seeking help (1\% as 29/2167). The screening instruments in this study were capable of helping identify nursing students at risk of poor mental health. Table 2 is the study outcomes of mental health risks and the treatment-seeking behaviours.

Cohen's kappa coefficients were calculated to measure the internal consistency between the five identified mental health risks. IRUHR and CSPQ-B appeared to have a lower internal consistency when compared with the other four risks. PPS based on the screening results of BQSPS and depression based on those of TDQ showed a consistency of 0.42 , and the consistency between HTA and depression read 0.36. Table 3 shows the internal consistency among mental health risks. 
Table 3 Internal consistency among mental health risks

\begin{tabular}{lrlll}
\hline Targeted risk & IRUHR & PPS & HTA & HSAGR \\
\hline PPS & $0.093^{\star}$ & & & \\
HTA & $0.108^{*}$ & $0.277^{\star}$ & & \\
HSAGR & $-0.004 \dagger$ & $0.013^{\star}$ & $0.041^{*}$ & \\
Depression & $0.103^{\star}$ & $0.422^{\star}$ & $0.358^{\star}$ & $0.019^{\star}$ \\
\hline
\end{tabular}

*The post hoc tests was performed using Bonferroni correction. The significant level of individual verification was calculated by the original significant level divided by the number of verifications. After the revision, $\alpha=\frac{0.05}{5}=0.01, \mathrm{p}<0.000001$.

$\mathrm{tp}=0.788$

HSAGR, high state anxiety and genetic risk; HTA, high trait anxiety; IRUHR, increased risk for ultra-high risk; PPS, putative prepsychosis states.

Correlations between the study screening instruments SPSS V.20.0 was used for two-tailed tests with $\alpha=0.050 .{ }^{30}$ The Pearson correlation coefficients were used to measure the correlations among the study mental health screening instruments (CSPQ-B, BQSPS, CMSTAI-Y and TDQ) and found the correlations among the screening tools range from 0.76 to 0.40 . A moderate correlation was observed between CSPQ-B and BQSPS $\mathrm{r}(2695)=0.68, \mathrm{p}<0.001$. The three subscales of CSP-Q (interpersonal, cognitiveperceptual and disorganisation) and the four subscales of BQSPS (interpersonal difficulty, subthreshold psychotic-like experiences, self-depreciation and negative symptoms) appeared to correlate to each other. CMSTAI-Y1 on state anxiety was moderately correlated to both CSPQ-B r
(2673) $=0.40, \quad \mathrm{p}<0.001$ and to BQSPS $\mathrm{r}(2685)=0.45$, $\mathrm{p}<0.001$. In addition, a strong correlation was found between CMSTAI-Y2 and CMSTAI-Y1 r $(2620)=0.76$, $\mathrm{p}<0.001$. TDQ for screening depression emerged to be moderately correlated to CSPQ-B $\mathrm{r}(2692)=0.54$, $\mathrm{p}<0.001$, to BQSPS $\mathrm{r}(2673)=0.63, \mathrm{p}<0.001$. to CMSTAI-Y1 $\mathrm{r}(2650)=0.58, \mathrm{p}<0.001$ and to CMSTAI-Y2 $\mathrm{r}(2639)=0.66$, $\mathrm{p}<0.001$. Table 4 shows the study results about correlations between the screening instruments.

\section{Anxiety and depression assessment results}

The mean scores of the participating nursing students read, respectively, 45.64 points $(\mathrm{SD}=8.38$ ) for CMSTAI-Y2, 40.32 points $(\mathrm{SD}=9.616)$ for CMSTAI-Y1 and 9.43 points $(\mathrm{SD}=8.20)$ for TDQ. The median scores were, respectively, 46 points for CMSTAI-Y2, 40 points for CMSTAI-Y1 and 7 points for TDQ. Around 127 (5\%) of the participants appeared to have severe anxiety based on the assessment by CMSTAI-Y2 ( $\geqq 60$ points), and 60 (2\%) by CMSTAI-Y1 ( $\geqq 60$ points). TDQ identified the participating nursing students with depression, including 244 (9\%) of the moderate depression ( $\geqq 19$ point) and $98(4 \%)$ of the students with major/severe depression ( $\geqq 29$ points). Please refer to table 5 for the screening results concerning anxiety and depression.

\section{DISCUSSION AND FINDINGS \\ Summary of findings}

We found that a total of $612(22 \%)$ of the participants were identified as having positive reactions among four

\begin{tabular}{|c|c|c|c|c|c|c|c|c|c|c|}
\hline Screening tool & CSPQ-B & BQSPS & CMSTAI-Y2 & CMSTAI-Y1 & $\begin{array}{l}\text { CSPQ- } \\
\text { B-I }\end{array}$ & $\begin{array}{l}\text { CSPQ- } \\
\text { B-C }\end{array}$ & $\begin{array}{l}\text { CSPQ- } \\
\text { B-D }\end{array}$ & BQSPS-I & $\begin{array}{l}\text { BQSPS- } \\
\text { SPE }\end{array}$ & BQSPS-S \\
\hline CMSTAI-Y2 & $0.465^{\star}$ & $0.588^{*}$ & & & & & & & & \\
\hline CMSTAI-Y1 & $0.395^{\star}$ & $0.454^{*}$ & $0.759^{\star}$ & & & & & & & \\
\hline CSPQ-B-D & & & & & $0.568^{\star}$ & $0.415^{\star}$ & & & & \\
\hline BQSPS-I & & & & & $0.702^{*}$ & $0.243^{*}$ & $0.422^{*}$ & & & \\
\hline BQSPS-SPE & & & & & $0.417^{\star}$ & $0.501^{*}$ & $0.398^{\star}$ & $0.393^{*}$ & & \\
\hline BQSPS-S & & & & & $0.391^{*}$ & $0.278^{*}$ & $0.341^{*}$ & $0.453^{*}$ & $0.369^{*}$ & \\
\hline
\end{tabular}

*The post hoc tests was performed using Bonferroni correction. The significant level of individual verification was calculated by the original significant level divided by the number of verifications. After the revision, $\alpha=\frac{0.05}{5}=0.01, \mathrm{p}<0.000001$.

BQSPS, Brief self-report Questionnaire for Screening Putative prepsychotic States; BQSPS-I, Brief self-report Questionnaire for Screening Putative prepsychotic States, Interpersonal difficulty/social anxiety subscale; BQSPS-N, Brief self-report Questionnaire for Screening Putative prepsychotic States, Negative symptoms subscale; BQSPS-S, Brief self-report Questionnaire for Screening Putative prepsychotic States, Self-depreciation subscale; BQSPS-SPE, Brief self-report Questionnaire for Screening Putative prepsychotic States, Subthreshold psychotic-like experiences subscale; CMSTAI-Y1, Chinese Mandarin State-Trait Anxiety Inventory Y1; CMSTAI-Y2, Chinese Mandarin State-Trait Anxiety Inventory Y2; CSPQ-B, Chinese Schizotypal Personality Questionnaire-Brief; CSPQ-B-C, Chinese Schizotypal Personality Questionnaire-Brief, Cognitive-perceptual subscale; CSPQ-B-D, Chinese Schizotypal Personality Questionnaire-Brief, Disorganisation subscale; CSPQ-B-I, Chinese Schizotypal Personality Questionnaire-Brief, Interpersonal subscale; TDQ, Taiwanese Depression Questionnaire. 


\begin{tabular}{|c|c|c|}
\hline Severity of anxiety/depression & $\begin{array}{l}\text { No of } \\
\text { students (n) }\end{array}$ & $\%$ \\
\hline CMSTAI-Y2 <40 no anxiety & 633 & 22.8 \\
\hline 40-59 Moderate anxiety & 2019 & 72.7 \\
\hline$\geqq 60$ Severe anxiety & 127 & 4.6 \\
\hline CMSTAI-Y1 $<40$ no anxiety & 1177 & 42.4 \\
\hline 40-59 Moderate anxiety & 1542 & 55.5 \\
\hline$\geqq 60$ Severe anxiety & 60 & 2.2 \\
\hline TDQ $<19$ no depression & 2437 & 87.7 \\
\hline 19-28 Moderate depression & 244 & 8.8 \\
\hline$\geqq 29$ Severe depression & 98 & 3.5 \\
\hline
\end{tabular}

CMSTAI-Y1, Chinese Mandarin State-Trait Anxiety Inventory Y1; CMSTAI-Y2, Chinese Mandarin State-Trait Anxiety Inventory Y2; TDQ, Taiwanese Depression Questionnaire.

psychiatric assessment tools on 5 identified mental health risks, which were indicated as the population having higher consistency of ultra-high risk with potential comorbidities of anxiety and depression. The participants who were identified to be positive on most of the psychological assessment tools were also reported as having higher percentage of seeking mental health assistance previously (4\%-17\%). Of the 69 students who had sought mental health assistance in the past, $40(58 \%)$ were identified as having mental health risks in this study, and 24 students had more than two risks. Those students were at higher risk of poor mental health and in a more urgent need of prioritised assistance in care and treatments.

The results of CSPQ-B and BQSPS on evaluating the risk and presymptoms of mental illness were quite different. There were 33 (1\%) IRUHR individuals identified by CSPQ-B and 446 (16\%) appeared to be positive by BQSPS. Out of 33 positive cases of CSPQ-B, up to 27 $(82 \%)$ were also identified as positive by BQSPS, which indicated that CSPQ-B has higher threshold in screening criteria. The previous systematic researches on mental illness risk screening had showed that different tools, screening threshold, may cause differences in screening results. Higher threshold can be used to reduce false positives. $^{32}$

The results showed that not only the scores of CSPQ-B, BQSPS, CMSTAI-Y and TDQ were moderately correlated with each other $(0.76$ to 0.40$)$; the five mental health risks were also overlapped as comorbidity. Twenty-three $(70 \%)$ cases with IRUHR also met the risk of depression, and 10 $(30 \%)$ cases were at high risk of HTA. For those who were in the PPS, $44 \%$ met the risk of depression and $21 \%$ were at high risk of HTA. These results are consistent with other studies which $75 \%$ of the cases with similar psychiatric experience have mental distress. ${ }^{33}$ Depressive disorders, fear and anxiety are the most common comorbidities in help-seeking prodromal risk syndrome. ${ }^{563435}$ Therefore, effectively dealing with depression to improve their responses to environmental stress and ambient stressors is very important. ${ }^{36} 37$

In this study, the score of the four mental health screening tools was significantly correlated with each other. The difference was found in the CSPQ-B score between male and female, which was different in our hypothesis. The agreement between the five definitions of high-risk mental state was determined using the kappa statistical analysis. The IRUHR identified by CSPQ-B had a lower consistency with other mental health risks, which showed that CSPQ-B not only has rigorous standards but also has good differentiating effect. Therefore, this study highly suggests that the implementation of CSPQ-B comparing to BQSPS is more suitable for future screening of high-risk mental state of nursing students.

\section{Further finding in context}

There were $1 \%$ of the subjects scored CSPQ-B $\geqq 17$ points in this study as IRUHR individuals, which was lower than other related studies $(3 \%-4 \%) .{ }^{21-23} 38$ Our average results of $\mathrm{CSPQ}-\mathrm{B}(\mathrm{M}=5.09, \mathrm{SD}=4.00)$ was similar to that of Compton $e t a l^{39}$ in the study of non-psychiatric control group, but lower than other studies $(\mathrm{M}=6.54, \mathrm{SD}=4.50) .{ }^{40}$ BQSPS $\geqq 8$ as PPS was $16 \%$, which was lower than other related studies $(21 \%-23 \%),{ }^{61}$ but higher than other studies $(9 \%-13 \%) .{ }^{57}$ The psychotic symptoms are closely related to age and population characteristics, the young age and the clinical help-seeking group had higher proportion of having mental health risk. ${ }^{32}$ In comparison with the above studies, the differences may be due to the broader age group, higher average age and general population rather than the clinical help-seeking group.

And also, among the questions of BQSPS, the result of the 15 th question that with direct relation to auditory hallucinations asked Do you hear some sounds, voices, or calls of your name when nobody is around you?'was scored 231 (8\%). A systematic review of all reported incidence and prevalence studies of population rates of subclinical psychotic experiences reveals a median prevalence rate of around $5 \%$ and a median incidence rate of around $3 \% .{ }^{42}$ When conducting a research using self-reported community screening survey based on general population rather than clinical help-seeking individuals, the issues of false positive must be highly valued. ${ }^{43}$ Due to the frequency of symptoms' onset, intensity and degree of intrusion to the cases, the mental illness might not be developed. Furthermore, the differences in evaluation methods such as self-report, clinical interview, lay-interview and so on might also cause differences in the final results. ${ }^{32}$ In the early detection of mental illness, the identification and confirmation of cases should be particularly strict and cautious in order to avoid the induced anxiety and fear towards social stigma. ${ }^{44}$

This study conducted self-reported survey on non-clinical help-seeking individuals without mental health clinical interviews. The issue of false positive was needed to take into consideration. In the determination of the risk of mental illness, diagnostic interviews require a lot of 
time and long-term professional training, and the cost is extremely high. ${ }^{45}$ Therefore, for large-scale community initial screening with simple questionnaires, the evaluation of mental symptoms, anxiety, depression and so on together with stricter threshold can be used to form a more rigorous assessment with lower cost for the health system. And those who have positive responses in different assessment tools should give priority to diagnostic evaluation.

\section{Interpretation and policy implications}

The previous studies had shown that mental health not only affected the personal and social adjustment and academic and clinical performance of the nursing students, ${ }^{13}$ it also caused medical errors ${ }^{46}$ and burnout. ${ }^{47} 48$ And the systematic research analysis had found that the majority of studies provided evidence that well-being in particular, poor wellbeing, as characterised by depression, anxiety, poor quality of life and stress, were found to be significantly associated with more self-reported errors, with a smaller number of studies showing an association of these factors with objective measures of error. ${ }^{47}$

Nursing students are valuable as the frontline and the basic labour force of the medical industry, and yet they are also vulnerable to the prodromal symptoms, anxiety and depression. The previous study had showed that the prodromal symptoms were closely related to anxiety and depression. ${ }^{49} 50$ However, another study on the systematic review of the early screening for the mental illness risk indicated that most of the study only conducted one assessment tools, and mostly not on nursing students. ${ }^{32}$

This study conducted four different psychiatric assessment tools, explored five identified mental health risks, and analysed the mental health risks of nursing students with both breadth and depth. The results showed that not only the scores of CSPQ-B, BQSPS, CMSTAI-Y and TDQ were moderately correlated with each other; the five mental health risks were also overlapped as comorbidity. These findings give an insight into the mental health of nursing students, these students are the future of the healthcare system and it is important that they are able to monitor their own mental health and seek treatment when needed. Educators on healthcare programmes need to ensure that a broad approach to education is fostered within their institutions to promote positive mental health among their students. The screening instruments adopted by this study were capable of serving as a workable model for early identification of nursing students at high risk of poor mental health, helping them to develop a stronger sensitivity to their mental health and assisting the faculty in providing early intervention to maintain their mental and overall well-being of the nursing students. The easy-to-use screening scales for assessing the risk to poor mental health among college nursing students is crucial importance to overall healthcare system.

\section{Study limitation}

This study conducted self-reported survey on non-clinical help-seeking individuals without mental health clinical interviews. The issue of false positive was needed to take into consideration during the initial screening of college students for identifying the high-risk mental state. Meanwhile, each subject received a total of 107 questions in five assessment tools in this study. The concentration of the subject might be affected. Therefore, questionnaire fatigue might also affect the results. ${ }^{51}$ Furthermore, the experience of some mental symptoms is not yet a predictor of future development of mental illness. Its role needs to be explored further.

\section{CONCLUSION}

This cross-sectional study assessed five identified mental health risks by using four psychiatric assessment tools on college nursing students. We found that $58 \%$ of those who sought assistance in the past were identified as having mental health risks in this study, and $35 \%$ had more than two risks. The subjects who were identified to be positive on most of the assessment tools were more likely to have sought mental health assistance previously. Moreover, those who scored positive on different assessment tools should give priority to diagnostic evaluation. The easy-to-use screening assessment tools can be adopted to assess the mental health state of nursing students who are the important assets of the healthcare industry. Ultimately, when assessing mental symptoms, anxiety, depression and so on simultaneously with stricter threshold as screening criteria, a more rigorous examination can be formed.

Future studies are recommended to focus on the developments of effective follow-up and intervention for nursing students at risk of mental health symptoms; the utilisations of professional training on the understanding of anxiety, depression and symptoms of ultra-high risk; and the strategies for encouraging students to seek clinical help and make use of related resources to maintain their mental well-being.

Acknowledgements We thank all participating nursing students and faculties of Hungkuang University.

Contributors Study design, data collection and data analysis were by D-YH, C-LT, $\mathrm{L}-\mathrm{CC}$ and $\mathrm{W}-\mathrm{FM}$. The manuscript preparation and critical revision of the manuscript were by D-YH, C-LT, L-CC and W-FM.

Funding The study was supported by research grants from China Medical University (CMU104-S-34, CMU107-BC-4) and Ministry of Science and Technology (MOST105-2314-B-039-038-MY2, MOST107-2314-B-039-041, MOST1072314-B-039-010), Taiwan.

Competing interests None declared.

Patient consent for publication Obtained.

Ethics approval The study was approved by the Institutional Review Board of Kuang Tien General Hospital, Taiwan (KTGH-10402).

Provenance and peer review Not commissioned; externally peer reviewed.

Data sharing statement These study data are deidentified participant data. The data that support the findings of this study are available beginning 12 months and ending 36 months following the article publication from the corresponding author, W-FM, upon reasonable request at Indaisy@mail.cmu.edu.tw.

Open access This is an open access article distributed in accordance with the Creative Commons Attribution Non Commercial (CC BY-NC 4.0) license, which permits others to distribute, remix, adapt, build upon this work non-commercially, and license their derivative works on different terms, provided the original work is properly cited, appropriate credit is given, any changes made indicated, and the use is non-commercial. See: http://creativecommons.org/licenses/by-nc/4.0/. 


\section{REFERENCES}

1. Kessler RC, Berglund P, Demler O, et al. Lifetime prevalence and age-of-onset distributions of DSM-IV disorders in the National Comorbidity Survey Replication. Arch Gen Psychiatry 2005;62:593-602.

2. Vanheusden $\mathrm{K}$, Mulder CL, van der Ende J, et al. Young adults face major barriers to seeking help from mental health services. Patient Educ Couns 2008;73:97-104.

3. Marshall M, Lewis S, Lockwood A, et al. Association between duration of untreated psychosis and outcome in cohorts of first-episode patients: a systematic review. Arch Gen Psychiatry 2005;62:975-83.

4. de Koning MB, Bloemen OJ, van Amelsvoort TA, et al. Early intervention in patients at ultra high risk of psychosis: benefits and risks. Acta Psychiatr Scand 2009;119:426-42.

5. Chen F, Wang L, Heeramun-Aubeeluck A, et al. Identification and characterization of college students with attenuated psychosis syndrome in China. Psychiatry Res 2014;216:346-50.

6. Kelleher I, Murtagh A, Molloy C, et al. Identification and characterization of prodromal risk syndromes in young adolescents in the community: a population-based clinical interview study. Schizophr Bull 2012;38:239-46.

7. Schultze-Lutter F, Michel C, Ruhrmann S, et al. Prevalence and clinical significance of DSM-5-attenuated psychosis syndrome in adolescents and young adults in the general population: the Bern Epidemiological At-Risk (BEAR) study. Schizophr Bull 2014:40:1499-508.

8. Yung AR, Phillips LJ, Yuen HP, et al. Psychosis prediction: 12-month follow up of a high-risk ("prodromal") group. Schizophr Res 2003;60:21-32.

9. McDonald M, Christoforidou E, Van Rijsbergen N, et al. Using Online Screening in the General Population to Detect Participants at Clinical High-Risk for Psychosis. Schizophr Bull 2018:1-10.

10. Simon AE, Velthorst $\mathrm{E}$, Nieman $\mathrm{DH}$, et al. Ultra high-risk state for psychosis and non-transition: a systematic review. Schizophr Res 2011;132:8-17.

11. Turner AP, Hammond CL, Gilchrist M, et al. Coventry university students' experience of mental health problems. Couns Psychol Q 2007;20:247-52.

12. Storrie K, Ahern K, Tuckett A. A systematic review: Students with mental health problems--a growing problem. Int J Nurs Pract 2010;16:1-6

13. Horgan A, Sweeney J, Behan L, et al. Depressive symptoms, college adjustment and peer support among undergraduate nursing and midwifery students. J Adv Nurs 2016;72:3081-92.

14. Horgan A, Kelly P, Goodwin J, et al. Depressive Symptoms and Suicidal Ideation among Irish Undergraduate College Students. Issues Ment Health Nurs 2018;39:575-84.

15. Ibrahim AK, Kelly SJ, Adams CE, et al. A systematic review of studies of depression prevalence in university students. J Psychiatr Res 2013;47:391-400.

16. Tung YJ, Lo KKH, Ho RCM, et al. Prevalence of depression among nursing students: A systematic review and meta-analysis. Nurse Educ Today 2018;63:119-29.

17. Lin SJ, Yen WJ, Lee S. The prevalence and related factors of depression in first year nursing students. Chung Shan Medical Journal;12011:61-9.

18. Xu Y, Chi X, Chen S, et al. Prevalence and correlates of depression among college nursing students in China. Nurse Educ Today 2014;34:e7-e12.

19. Chernomas WM, Shapiro C. Stress, depression, and anxiety among undergraduate nursing students. Int J Nurs Educ Scholarsh 2013;10:255-66

20. Keshavan MS, DeLisi LE, Seidman LJ. Early and broadly defined psychosis risk mental states. Schizophr Res 2011;126(1-3):1-10.

21. Raine A, Benishay D. The SPQ-B: a brief screening instrument for schizotypal personality disorder. J Pers Disord 1995;9:346-55.

22. Compton MT, Goulding SM, Bakeman R, et al. An examination of the factorial structure of the Schizotypal Personality QuestionnaireBrief (SPQ-B) among undergraduate students. Schizophr Res 2009;115:286-9.

23. Ma WF, Wu PL, Yang SJ, et al. Sensitivity and specificity of the Chinese version of the Schizotypal Personality Questionnaire-Brief for identifying undergraduate students susceptible to psychosis. Int J Nurs Stud 2010;47:1535-44.

24. Liu CC, Tien YJ, Chen CH, et al. Development of a brief self-report questionnaire for screening putative pre-psychotic states. Schizophr Res 2013;143:32-7.
25. Nuñez D, Arias VB, Campos S. The Reliability and Validity of Liu's Self-Report Questionnaire for Screening Putative Pre-Psychotic States (BQSPS) in Adolescents. PLoS One 2016;14:1-11.

26. Demmin DL, DeVylder JE, Hilimire MR. Screening for sub-threshold psychotic experiences and perceived need for psychological services. Early Interv Psychiatry 2017;11:139-46.

27. Spielberger CD. State-Trait Anxiety Inventory. CA: Consulting Psychologists Press1983.

28. Ma WF, Liu YC, Chen YF, et al. Evaluation of psychometric properties of the Chinese Mandarin version State-Trait Anxiety Inventory $Y$ form in Taiwanese outpatients with anxiety disorders. J Psychiatr Ment Health Nurs 2013;20:499-507.

29. Lee $Y$, Yang MJ, Lai TJ, et al. Development of the Taiwanese Depression Questionnaire. Chang Gung Med J 2000;23:688-94.

30. Lee AK. A simple guide to IBM SPSS statistics: for version 20.0 Australia: Wadsworth Cengage Learning, 2013.

31. Faul F, Erdfelder E, Buchner A, et al. Statistical power analyses using $\mathrm{G}^{*}$ Power 3.1: tests for correlation and regression analyses. Behav Res Methods 2009;41:1149-60.

32. Kline E, Schiffman J. Psychosis risk screening: a systematic review. Schizophr Res 2014;158(1-3):11-18.

33. Kelleher I, Wigman JT, Harley M, et al. Psychotic experiences in the population: Association with functioning and mental distress. Schizophr Res 2015;165:9-14.

34. Rosen JL, Miller TJ, D'Andrea JT, et al. Comorbid diagnoses in patients meeting criteria for the schizophrenia prodrome. Schizophr Res 2006;85:124-31.

35. Svirskis T, Korkeila J, Heinimaa M, et al. Axis-I disorders and vulnerability to psychosis. Schizophr Res 2005;75:439-46.

36. Cantor-Graae E. The contribution of social factors to the development of schizophrenia: a review of recent findings. Can J Psychiatry 2007;52:277-86.

37. Fusar-Poli P, Valmaggia L, McGuire P. Can antidepressants prevent psychosis? Lancet 2007;370:1746-8.

38. Kline E, Wilson C, Ereshefsky S, et al. Schizotypy, psychotic-like experiences and distress: an interaction model. Psychiatry Res 2012;200(2-3):647-51.

39. Compton MT, Chien VH, Bollini AM. Psychometric properties of the Brief Version of the Schizotypal Personality Questionnaire in relatives of patients with schizophrenia-spectrum disorders and nonpsychiatric controls. Schizophr Res 2007;91:122-31.

40. Fonseca-Pedrero E, Ortuño-Sierra J, Lucas-Molina B, et al. Brief assessment of schizotypal traits: A multinational study. Schizophr Res 2017;197:182-91.

41. Schimmelmann BG, Michel C, Schaffner N, et al. What percentage of people in the general population satisfies the current clinical at-risk criteria of psychosis? Schizophr Res 2011;125:99-100.

42. van Os J, Linscott RJ, Myin-Germeys I, et al. A systematic review and meta-analysis of the psychosis continuum: evidence for a psychosis proneness-persistence-impairment model of psychotic disorder. Psychol Med 2009;39:179-95

43. Hanssen MS, Bijl RV, Vollebergh W, et al. Self-reported psychotic experiences in the general population: a valid screening tool for DSM-III-R psychotic disorders? Acta Psychiatr Scand 2003;107:369-77.

44. Correll CU, Hauser M, Auther AM, et al. Research in people with psychosis risk syndrome: a review of the current evidence and future directions. J Child Psychol Psychiatry 2010;51:390-431.

45. Kline E, Thompson E, Demro C, et al. Longitudinal validation of psychosis risk screening tools. Schizophr Res 2015;165:116-22.

46. Melnyk BM, Orsolini L, Tan A, et al. A National Study Links Nurses' Physical and Mental Health to Medical Errors and Perceived Worksite Wellness. J Occup Environ Med 2018;60:126-31.

47. Hall LH, Johnson J, Watt I, et al. Healthcare staff wellbeing burnout, and patient safety: a systematic review. PLoS One 2016;11:e0159015.

48. Johnson J, Hall LH, Berzins K, et al. Mental healthcare staff wellbeing and burnout: a narrative review of trends, causes, implications, and recommendations for future interventions. Int J Ment Health Nurs 2018;27:20-32.

49. Rapado-Castro M, McGorry PD, Yung A, et al. Sources of clinical distress in young people at ultra high risk of psychosis. Schizophr Res 2015;165:15-21.

50. Cornblatt BA, Lencz T, Smith CW, et al. The schizophrenia prodrome revisited: a neurodevelopmental perspective. Schizophr Bull 2003;29:633-51

51. Rolstad S, Adler J, Rydén A. Response burden and questionnaire length: is shorter better? A review and meta-analysis. Value Health 2011;14:1101-8 